\title{
Temperature Dependent Magnetic Susceptibility and Equilibrium Studies in Solution: Evans's Method Dipesh Ghosh
}

Department of Chemistry, Vivekananda Mission Mahavidyalaya, Haldia, 721645, West Bengal, India

Received: November 23, 2016 | Accepted: April 28, 2017 | Published online: April 28, 2017

\begin{abstract}
The chemical shift in a solvent caused by the presence of a paramagnetic species and the temperature dependence of the chemical shift difference to determine the temperature dependent magnetic susceptibility can be studied using Evans method. The method is very useful because a common NMR instrument, easily accessible in a department of chemistry, allows the accurate measurement of paramagnetic susceptibilities. The present review highlights planar $(\mathrm{S}=0)$ and octahedral $(\mathrm{S}=1)$ forms of $\mathrm{Ni}(\mathrm{II})$ complexes are in equilibrium and has been followed in the temperature range $298-338 \mathrm{~K}$ by ${ }^{1} \mathrm{H}$ NMR technique using the protocol of Evans's method.
\end{abstract}

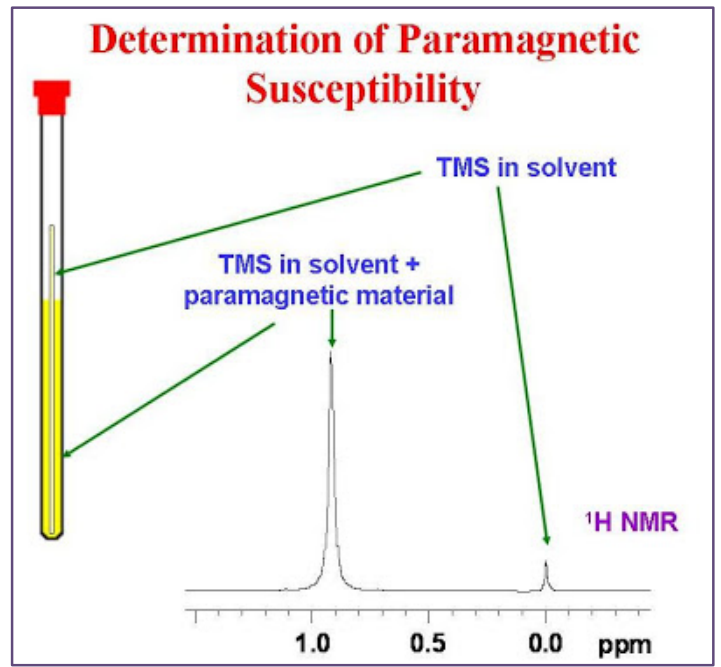

Keywords: Nickel(II) complexes, octahedral and planar forms, equilibrium in solution, Evans's method,

\section{Introduction}

The chemical shift of a magnetic nucleus is a complex function of many variables and these have been discussed adequately in several text books dealing with the principles of $\mathrm{nmr}$ spectroscopy. One important factor is the magnetic susceptibility. The position of a line in the proton resonance spectrum of a molecule depends on the bulk susceptibility of the medium in which the resonating nuclei are immersed. Chemical shift of a specific type of protons in a solvent and the change of this shift when the paramagnetic species is added was observed. Let $\Delta \kappa$ is the change in volume susceptibility. The difference in the magnetic resonance absorption of the protons in the two solutions caused by the paramagnetic substances are given by the theoretical expression ${ }^{1}$

$$
\frac{\Delta H}{H}=\left(\frac{2 \pi}{3}\right) \Delta \kappa
$$

For aqueous solutions of paramagnetic substances about $2 \%$ of t-butyl alcohol is incorporated as an inert reference substance, and a capillary containing the same concentration of t-butyl alcohol in water is also placed in the nuclear magnetic resonance tube which is spun during the measurement. (The change in the susceptibility of the dissolved compound caused by the t-butyl alcohol will normally be completely negligible.) Two resonance lines will normally be obtained from the methyl protons of the t-butyl alcohol in the two solutions owing to the difference in their volume susceptibilities, with the line from the more paramagnetic solution lying at higher frequencies. Acetone or dioxan can also be used in place of t-butyl alcohol or, for non-aqueous solutions, cyclohexane or tetramethylsilane. Alternatively a 


\section{Prayogik Rasayan}

resonance line of the organic solvent itself can be used as a reference, provided there is no interaction with the solute. Equation (1) may be restated in terms of more commonly used frequency separation $(\Delta f / f$ replacing $\Delta H / H)$ and by converting to mass susceptibility to give the following relation

$$
\chi_{g}=\frac{3 \Delta f}{2 \pi f m}+\chi_{0}
$$

where $\chi_{g}=\kappa / m$ is the mass susceptibility of the dissolved paramagnetic substance, where $\Delta f$ is the frequency separation between the two lines in cycles/sec (hertz), $f$ is the frequency at which the proton resonances are being studied, in cycles/sec, $m$ is the mass of substance contained in $1 \mathrm{ml}$. of solution, $\chi_{0}$ is the mass susceptibility of the solvent $\left(-0.72 \times 10^{-6}\right.$ for dilute t-butyl alcohol solutions). A correction term has been suggested by Evans to take into account any difference in density of the pure solvent, $d_{o}$, and that of the solution $d_{s}$, yielding

$z_{g}=\frac{3 \Delta f}{2 \pi f m}+z_{0}+\frac{\chi_{0}\left(d_{0}-d_{s}\right)}{m}$

For highly paramagnetic substances the last term can often be neglected without serious error, the details of which are described elsewhere. ${ }^{2}$ The determination of the mass susceptibility of a paramagnetic substance in solution is made, therefore, by measuring the difference in the chemical shift of some proton in the pure solvent and in a solution containing the paramagnetic substance of known concentration. The value of $\chi_{o}$ may be obtained by summing the atomic susceptibilities of the substituent atoms of the solvent (including contributions from any constituent effects, e.g., $\mathrm{C}=\mathrm{C}$ ) and dividing this sum by the molecular weight of the solvent. The atomic susceptibilities are available in various literature. The mass susceptibility, $\chi_{g}$, which results from eqn. (2) may be converted into molar susceptibility, $\chi_{M}$ ', by multiplying $\chi_{9}$, by the molecular weight of the complex. Then $\chi_{M}{ }^{\prime}$ must be corrected for the presence of the diamagnetic contribution from the ligand atoms. This is done by simply summing the diamagnetic contribution of each ligand atom and groups of atoms and adding the sum to the susceptibility of the complex to give the corrected molar susceptibility $\chi_{M}$. This is related directly to the magnetic moment by eqn. (4)

$$
\mu=2.84 \sqrt{\chi_{M} T}
$$

The octahedral-square-planar equilibrium in solution can be monitored by susceptibility $\left(\chi_{\mathrm{g}}\right)$ measurement at variable temperatures following Evans's method. Since one component of this equilibrium is a paramagnetic species while the other is a diamagnetic one, this offers an opportunity $^{2,3}$ to follow the above equilibrium by magnetic measurements in solution using ${ }^{1} \mathrm{H}$ NMR technique as proposed by Evans. ${ }^{1}$

\section{Equilibrium Studies}

The possibility of the existence of an equilibrium in solution between the octahedral and planar forms of nickel-(II) complexes was reported for the first time with some sterically constrained ligands, viz. stilbenediamine (stien) by Lifschitz et al. ${ }^{4,5}$ Over the years, several other such equilibria of two different kind (Scheme 1) have been reported using macrocyclic ${ }^{6-13}$ and open chain polyamines and Schiff base ligands. ${ }^{3,14-16}$ Usually square-planar species NiL of macrocyclic ligands L (Scheme 1A) generate the octahedral counterpart $\mathrm{NiLX}_{2}^{3,6-14}$ axial attachment of ligand $\mathrm{X}(\mathrm{X}=$ anions or solvent molecules, predominantly water). The second type of equilibrium (Scheme 1B), on the other hand, involves flexidentate ligands ( $\left.\mathrm{L}^{\prime}\right)$ which form bis complexes of composition $\mathrm{Ni}\left(\mathrm{L}^{\prime}\right)_{2}$. At lower temperatures, these ligands bind nickel(II) in tridentate fashion to render octahedral geometry which reduces to a planar one at elevated temperatures due to steric constraints of the associated ligands. ${ }^{15,16}$ Reports have been made on $\mathrm{pH}$-dependent reversible translocation 


\section{Prayogik Rasayan}

of $\mathrm{Ni}$ (II) ion from octahedral to the square-planar site in ditopic ligand systems. ${ }^{17}$

Scheme 1

A.

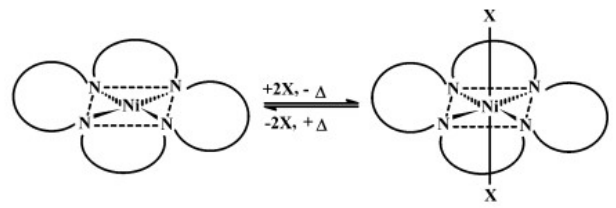

B.

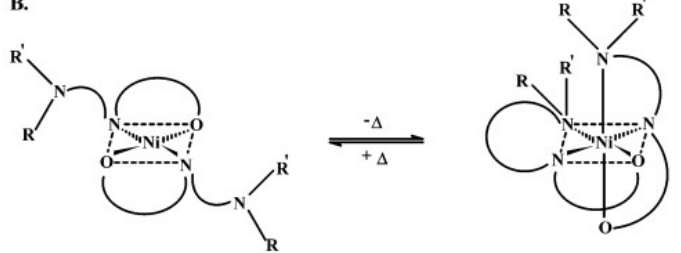

(Reprinted with permission from ref 18 . Copyright ACS)

Most of these equilibrium studies were followed by spectroscopic techniques where the squareplanar-octahedral equilibrium in solution can be followed in detail by ${ }^{1} \mathrm{H}$ NMR and electronic spectroscopies.

\subsection{Our Contribution}

The common strategy to obtain both square planar and octahedral mononuclear Ni(II) complexes showing equilibrium in solution is to use Schiff bases prepared from the condensation of amino ethyl piperazine with aromatic aldehydes or its derivatives, 1-phenyl-1,3butanedione/acetyl acetone in 1:1 ratio. The piperazine arm of these ligands can, in principle, have both boat and chair conformations that force these molecules to display ambidentate ligation behavior, leading to both octahedral and square-planar geometry for the $\mathrm{Ni}$ (II) complexes. By ${ }^{1} \mathrm{H}$ NMR technique using the protocol of Evans's method both these planar $(\mathrm{S}=0)$ and octahedral $(\mathrm{S}=1)$ equilibrium can be followed in a particular range of temperature and the equilibrium constant $K_{\mathrm{eq}}$ as well as other thermodynamic parameters can be explored.

We have reported ${ }^{18}$ structural and equilibrium studies of nickel(II) complexes of flexidentate 5-substituted salicylaldimino Schiff base ligands based on 1-(2-aminoethyl)piperazine. The piperazinyl arm of these ligands can in principle have both boat and chair conformations that allow the ligands to bind the $\mathrm{Ni}(\mathrm{II})$ center in an ambidentate manner, forming square-planar and/or octahedral complexes
(Figure 1). Also, this is one of the rare examples ${ }^{13,19}$ where both forms involved in equilibrium have been isolated in the solid state and characterized crystallographically. In solution, these compounds are in equilibrium, which is unique in the sense that both solvation and the change of ligand denticity are simultaneously in operation here.

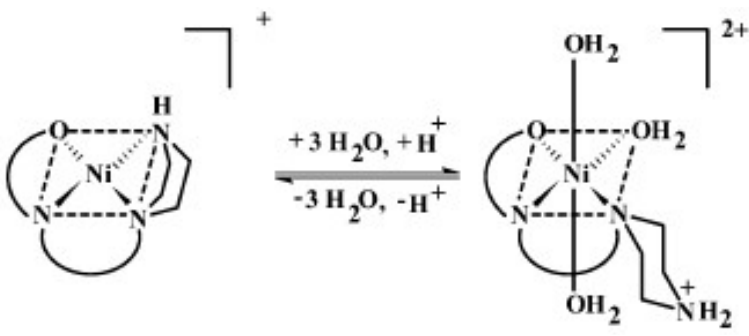

Figure 1. Square-planar-octahedral equilibrium. (Reprinted with permission from ref 18. Copyright ACS)

\section{Methodology of Magnetic Susceptibility Measurement}

The octahedral-square-planar equilibrium in solution was monitored by susceptibility $\left(\chi_{\mathrm{g}}\right)$ measurement at variable temperatures following Evans's method. ${ }^{1}$ The working solution in $\mathrm{D}_{2} \mathrm{O}$ containing 2\% tert-butyl alcohol as a reference compound was prepared by dissolving $0.040 \mathrm{~g}$ of paramagnetic compound per milliliter of the solvent. The solution was taken in a NMR tube. The same combination of solvent ( $2 \%$ tert-butyl alcohol in $\mathrm{D}_{2} \mathrm{O}$ ) was also used as external standard and taken in a capillary tube of $2 \mathrm{~mm}$ outer diameter. The latter was placed inside the NMR tube, and the combination was used for $\Delta f$ measurements in the temperature range 298-338 K, using a Bruker DPX 300 NMR spectrometer, operated at $300 \mathrm{MHz}$ frequency. The ${ }^{1} \mathrm{H}$ NMR spectra of the reference compound in the two coaxial tubes, due to the difference in their volume susceptibilities, exhibit chemical-shift differences $(\Delta f)$, which are measured (in hertz) and used to calculate the mass susceptibility $\left(\chi_{\mathrm{g}}\right)$ of the dissolved paramagnetic molecule from equation 3 .

A gradual decrease in the magnetic moment value with the rise in temperature is a clear indication of the transformation of paramagnetic 


\section{Prayogik Rasayan}

into the diamagnetic species. The percentage of diamagnetic species present in solution has been calculated using ${ }^{2,15}$

$$
\% \text { diamagnetic species }=100\left[1-\mu^{2} /(\mathrm{X})^{2}\right]
$$

where $\mu$ is the magnetic moment of the solution at any particular temperature and $\mathrm{X} \mu_{B}$ is the magnetic moment of pure paramagnetic species in the solid state. The equilibrium constant $K_{\text {eq }}$ is defined as $K_{\text {eq }}=$ [octahedral]/[planar]. Since the equilibrium is $\mathrm{pH}$ dependent, $K_{\text {eq }}$ here is a composite parameter that includes the contribution due to temperature dependence of $\mathrm{pH}$. A plot of $\log K_{\mathrm{eq}}$ versus $1 / T$ is linear giving $\Delta H^{\circ}$ from the leastsquares slope. The values of $\Delta S^{\circ}$ obtained at each temperature from the equation $\Delta G^{\circ}=\Delta H^{\circ}-T \Delta S^{\circ}$

\section{Conclusions}

This review has been concerned mainly with application of the Evans method for temperature dependent studies of paramagnetic susceptibility and equilibria. The Evans NMR method is helpful for determination of effective magnetic moments of complexes and for quantitative study of spin-state equilibrium. It is hoped that this short review will draw attention of the students to perform hands-on experiments.

\section{Acknowledgements}

I am thankful to Professor Muktimoy Chaudhury for his constant encouragement. Also thanks to Dr. Suman Mukhopadhyay for samples of $\mathrm{Ni}(\mathrm{II})$ complexes and NMR experiments. Financial support received from UGC, New Delhi is gratefully acknowledged.

\section{Author Information}

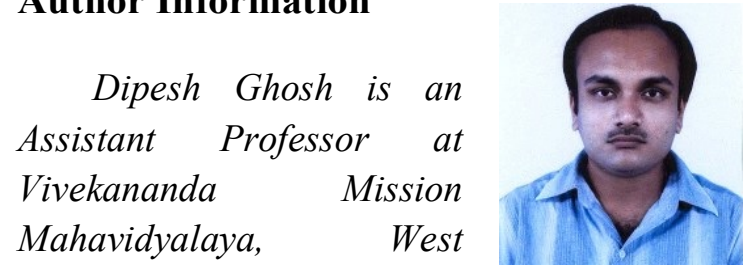

Bengal, India. He received his B.Sc. Hons. in Chemistry (1995) from the Ramakrishna Mission Vidyamandira, University of Calcutta. After being awarded M. Sc. Degree (1997, $5^{\text {th }}$ rank) from the University of calcutta, he joined the Department of Inorganic Chemistry, Indian Association for the Cultivation of Science, Jadavpur in for his doctoral research under the supervision of Prof. Muktimoy Chaudhury. He also awarded lectureship by UGC-CSIR in 2000. He received his $P h$. D. degree from the same institute in 2004. His areas of specialization have included bio-inspired inorganic chemistry, model compounds, X-ray crystallography, selfassembled receptors for ions and biomolecules. Prof. Ghosh did his postdoctoral work abroad of which most recent one at the University of Sheffield, Sheffield, UK (2007-2008), under the supervision of Prof. Jim A. Thomas. His regular teaching responsibilities include advanced inorganic chemistry and bioinorganic chemistry.

\section{References}

1. Evans, D. F. J. Chem. Soc. 1959, 2003.

2. Crawford, T. H.; Swanson, J. J. Chem. Educ. 1971, 48, 382.

3. Evans, D. F.; Missen, P. H. J. Chem. Soc., Dalton Trans. 1985, 1451.

4. Lifschitz, I.; Bos, J. G.; Dijkema, K. M. Z. Anorg. Allg. Chem. 1939, 24, 97.

5. Lifschitz, I.; Bos, J. G. Recl. Tra V . Chim. 1940, 5, 407.

6. Fabbrizzi, L.; Paoletti, P.; Clay, R. M. Inorg. Chem. 1978, 17, 1042.

7. Sabatini, L.; Fabbrizzi, L. Inorg. Chem. 1979, 18, 438.

8. Swisher, R. G.; Dayhuff, J. P.; Stuehr, D. J.; Blinn, E. L. Inorg. Chem. 1980, 19, 1336.

9. Hay, R. W.; Bembi, R.; Sommerville, W. Inorg. Chim. Acta 1982, 59, 147.

10. Steenland, M. W. A.; Dierck, I.; Herman, G. G.; Devreese, B.; Lippens, W.; Van Beeumen, J.; Goeminne, A. M. J. Chem. Soc., Dalton Trans. 1997, 3637.

11. Sakata, K.; Wada, S.; Sato, N.; Kurisu, M.; Hashimoto, M.; Kato, Y. Inorg. Chim. Acta 1986, 119, 111 .

12. Bembi, R.; Bhardwarj, V. K.; Singh, R.; Singh, R.; Taneja, K.; Aftab, S. Inorg. Chem. 1984, 23, 4153 .

13. Hay, R. W.; Jeragh, B.; Ferguson, G.; Kaitner, B.; Ruhl, B. L. J. Chem. Soc., Dalton Trans. 1982, 1531. 


\section{Prayogik Rasayan}

14. Anichini, A.; Fabbrizzi, L.; Paoletti, P. Inorg. Chim. Acta 1977, 24, L21.

15. Sacconi, L.; Nannelli, P.; Nardi, N.; Campigli, U. Inorg. Chem. 1965, 4, 943.

16. Sacconi, L.; Nardi, N.; Zanobini, F. Inorg. Chem. 1966, 5, 1872.
17. Amendola, V.; Fabbrizzi, L.; Mangano, C.; Pallavicini, P. Acc. Chem. Res. 2001, 34, 488.

18. Mukhopadhyay, S.; Mandal, D.; Ghosh, D.; Goldberg, I.; Chaudhury, M. Inorg. Chem. 2003, 42, 8439 .

19. Nyburg, S. C.; Wood, J. S. Inorg. Chem. 1964, 3, 468 . 